\title{
Energy Efficient Routing Protocol in Wireless Sensor Network: A Literature
}

\author{
Sarang Kharpate \\ Research Scholar \\ Dept. of ECE \\ T.I.E.I.T, Bhopal
}

\author{
Neelesh Gupta \\ H.O.D \\ Dept. of ECE \\ T.I.E.I.T, Bhopal
}

\author{
Neetu Sharma \\ Assistant Professor \\ Dept. of ECE \\ T.I.E.I.T, Bhopal
}

\begin{abstract}
Wireless sensor network gaining popularity due to its three most efficient features such as it can be access from anywhere, anytime and any person and have increased enormously in modern time due to development in MicroElectro-Mechanical Systems (MEMS) technology. Due to these technical improvements in wireless communication it offers less cost, sensor nodes and less power in wireless sensor network. The major issue of such network is routing in network layer because the radio transmission and reception consumes more power and energy. The energy saving becomes great deal nowadays because sensor nodes are battery operated device and its life time can be expanded by minimizing the energy consumption at each and energy layer of the network during the transmission. So the selection of routing mechanism is very important for the delivery of packets. Lots of work has been done to enhance the battery life time and minimizing the energy consumption. In this paper, a literature about the energy efficient routing in wireless sensor network introduced and different energy efficient routing techniques with their merits and demerits.
\end{abstract}

\section{Keywords}

Energy Efficiency, Routing Protocol, Wireless Sensor Network, Battery Lifetime, Micro-Electrical Mechanical System.

\section{INTRODUCTION}

Wireless Sensor Networks are turning into a requirement for the humanity because of the development in Micro-ElectroMechanical Systems (MEMS) technology. A processing device, sensor or motes in wireless sensor networks can accumulate information, handle it and transmit it to another device. Other device total the information acquired in a manner that it is understandable to the people. Wireless Sensor Networks (WSNs) is defined as an aggregation of a large number of sensor nodes which are densely deployed either inside a physical occurrence or very close to it [1]. Sensors are small device which monitor different conditions like temperature, clamminess, weight and so forth and later change over it into electrical flag. These sensor devices convey either straightforwardly to the Base Station (BS) or among each other. Every node henceforth requires a power source which can give a node utmost life notwithstanding its little size. The self-sorting out ability of sensor nodes gives a few difficulties among analysts to outlining the system conventions. The correspondence engineering of WSN comprises of sensor nodes scattered in a sensor field with each of these nodes equipped for gathering and directing information back to sink and the end clients as in Fig. 1. The correspondence convention have five standard layers: application layer, transport layer, arrange layer, information connect layer, physical layer and three administration planes: control administration plane, mobility management plane, and task management plane [2].

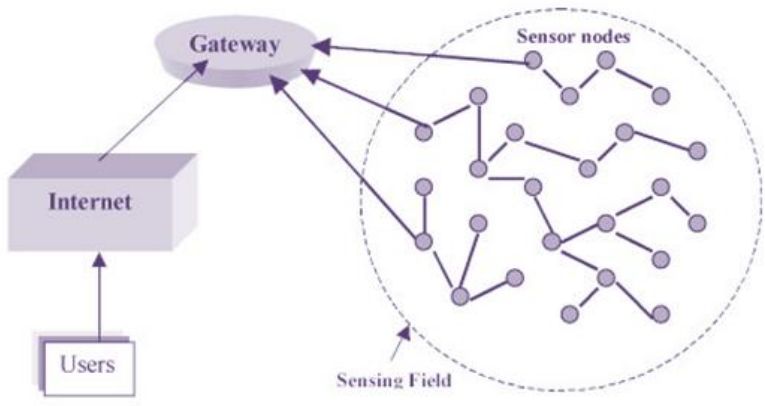

Fig.1. Wireless Sensor Network Architecture

The hardware architecture of a sensor node appeared in Fig.2 comprises of four parts: Sensing, Processing, Transmitter/Receiver and a Power Unit. They may likewise have location discovering framework and a mobilizer relying upon the applications [2]. A power generator might be available as an outer power provider. The real sympathy toward researchers and analysts is the power unit. To advance life time of node, calculations and conventions that make most extreme yield with constrained power assets ought to be outlined. WSN applications are basically delegated checking and following as appeared in the Fig 3. The potential applications incorporate military, detecting, air traffic control, movement observation, mechanical and producing mechanization, environment, wellbeing, home and other business territories. The outline of WSN is impacted by variables like adaptation to internal failure, versatility, working environment, equipment requirements, control and so forth and depends altogether on the application.

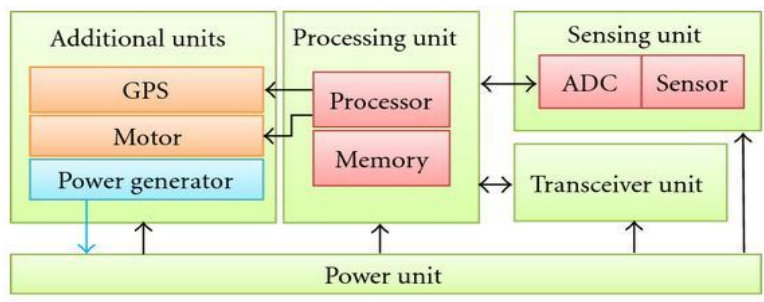

GPS: global positioning system

ADC: analog to digital converter

\section{Fig.2. Hardware Architecture}

The network layer handles directing and points in amplifying the lifetime by discovering courses for energy effective and reliable route foundation for data transmission from sensor nodes to sink. Numerous routing protocols have been proposed so as to route packets productively. The outline of 
routing protocols is additionally influenced by different elements, for example, deployment, energy utilization, security and so forth. Analysts subsequently concentrate more on outlining energy effective protocols. In this paper, we presents literature study about the former work done for the energy efficient routing and various technique with their merits and demerits.

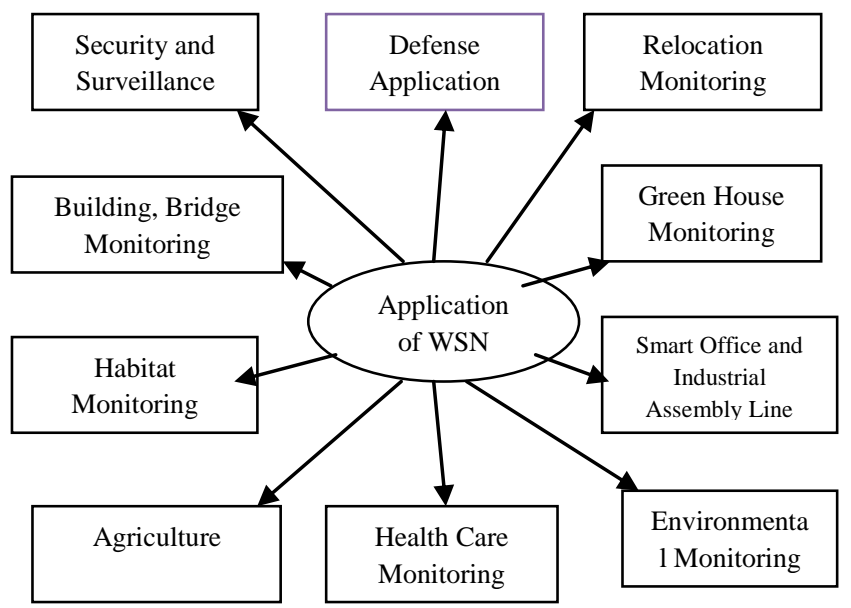

Fig.3: Wireless Sensor Network Application

The organization of remaining section of the paper is done as follows: Section II describes the classification of routing protocol for wireless sensor network. In section III describes the literature work for the efficient routing. Different energy efficient routing technique is discussed in section IV and last section gives overall conclusion of the paper with future work.

\section{CLASSIFICATION OF ROUTING PROTOCOL}

Routing in WSN differs from conventional routing .There is no infrastructure, wireless links are unreliable, sensor nodes may fail , and routing protocols have to meet strict energy saving requirements. Many routing algorithms were developed for wireless networks. When sensor nodes are static, it is preferable to have table driven routing protocols rather than using reactive protocols. a significant amount of energy is used in route discovery and setup of reactive protocols. All major routing protocols classified into seven main categories shown below:

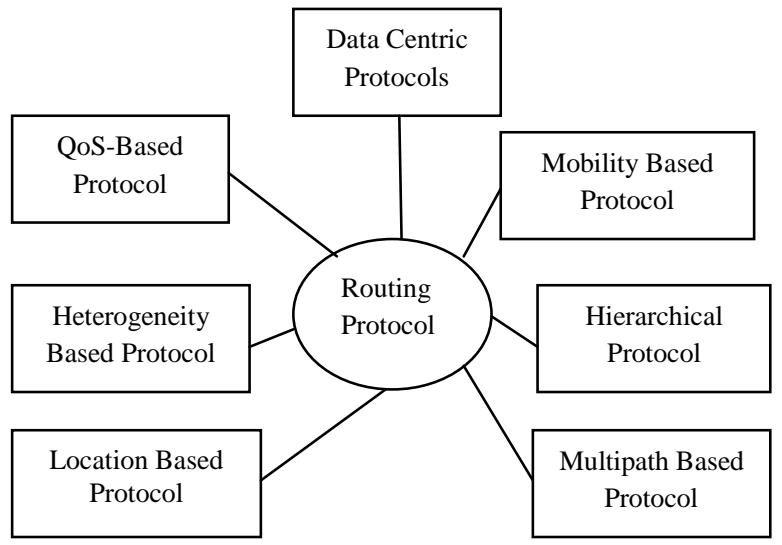

Fig.4: Classification of Routing Protocol in WSN

\subsection{Location Based Routing}

The location information based routing protocol uses location information to guide routing discovery and maintenance as well as data forwarding, enabling directional transmission of the information and avoiding information flooding in the entire network. Location information is needed in order to calculate the distance between two particular nodes so that energy consumption can be estimated [3].

\subsection{Hierarchical Routing Protocol}

Clustering is an energy efficient communication protocol that can be used by the sensors to report their sensed data to the sink. Hierarchical routing is to efficiently maintain the energy consumption of network. This provides inherent optimization capabilities at the cluster heads. A network is composed of several clusters [4]. Each cluster is managed by a special node, called cluster head, which is responsible for coordinating the data transmission activities of all sensors in its cluster. There are different hierarchical routing protocols such as: PEGASIS, TEEN, HEED and LEACH etc.

\subsection{Data-Centric Protocol}

Data centric protocol different from traditional address centric protocols in the data they carry. While in ad hoc networks individual data items are important, in sensor networks it is the aggregate data carried in the data rather than the actual data. In data centric routing, the end nodes, the sensors themselves, are less important than data itself. The sink sends queries to certain regions and waits for data from the sensors located in a selected region. Data centric protocols are classified in to nine categories of routing protocols are as follows: SPIN, DD, RR, MCFA, GBR, IDSQ, CADR, COUGAR, ACQUIRE, EAR [5].

\subsection{Multipath Routing Protocol}

Some Routing protocol are flexible multipath are used instead of single path. In case a path is down, an alternative path is directly chosen to deliver the data from source to destination. However, it may be energy consuming since these alternative paths are kept alive by sending periodic packets. Reliability is insured in the scheme. Examples: Directed Diffusion, Multi path and Multi SPEED (MMSPEED).[7]

\subsection{Quality of Service}

The network application business and its functionalities prompt the need for ensuring a QoS (Quality of Service) in the data exchange. In particular, effective sample rate, delay bounded and temporary precision are often required. Satisfying them is not possible for all the routing protocols as the demands may be opposite to the protocol principles. For instance, a routing protocol could be designed to extend the network lifetime while an application may demand an effective sample rate which forces periodic transmissions and, in turn, periodic energy consumptions. Figure 5 shows the relation of QoS and its dependence to the routing protocol goal and to the routing protocol strategy.[6]

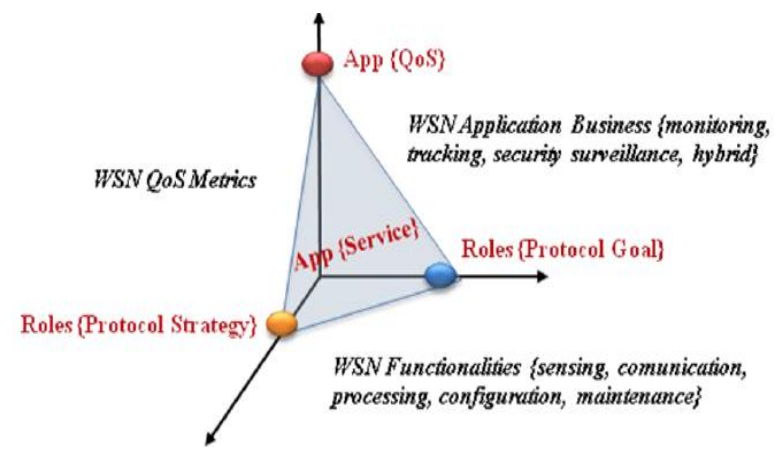

Fig.5: Relation of QoS and Routing Protocol 


\subsection{Heterogeneity Based Protocol}

In a heterogeneous wireless sensor network, higher energy nodes can become cluster heads more times than the nodes with low energies. This means that creation of cluster heads and assigning tasks to them can greatly contribute to energy efficiency which results in increase in stability and network lifetime.

\section{RELATED WORK}

The Wireless sensor network is the collection of sensor nodes which is the battery oriented and during the transmission and reception of the packet energy consumption is more. So the efficient routing selection becomes essential. Lots of work has been done in this area to minimize the energy consumption. This section of the paper describes the former work done in the field of energy efficient routing.

Kwon and Shroff[8]developed an energy-efficient routing scheme that takes into account the interference created by existing flows in the network. The routing scheme chooses a route such that the network expends the minimum energy satisfying with the minimum constraints of flows. Unlike previous works, we explicitly study the impact of routing a new flow on the energy consumption of the network. Under certain assumptions on how links are scheduled, we can show that our proposed algorithm is asymptotically (in time) optimal in terms of minimizing the average energy consumption. We also develop a distributed version of the algorithm. Our algorithm automatically detours around a congested area in the network, which helps mitigate network congestion and improve overall network performance. Using simulations, they showed that the routes chosen by our algorithm (centralized and distributed) are more energy efficient than the state of the art.

Mao et al. [9]proposed routing algorithm uses the proximity approach to find the appropriate set of nodes for transmission, thus, improving lifetime and resolving routing loop issues. The effectiveness of the proposed Proximity Based Energy Efficient Routing (PEER) is demonstrated in as gains attained in terms of improved lifetime, and energy consumption. Analyses proved the effectiveness of the proposed PEER algorithm in comparison with RDSR and RIDSR algorithm in terms of significant gains attained for energy consumption and number of iterations.

Razaque et al. [10] introduced PEGASIS-LEACH (PLEACH), a near optimal cluster-based chain protocol that is an improvement over PEGASIS and LEACH both. This protocol uses an energy efficient routing algorithm to transfer the data in WSN. To validate the energy effectiveness of PLEACH, we simulate the performance using Network Simulator (NS2) and MATLAB. Based on the simulation results, they determined that P-LEACH performs better than LEACH and PEGAIS in terms of energy and lifetime of the network. The simulation results validate that our proposed approach could extend the network for WSNs applications.

Handy et al.[11] focus on reduction in power consumption of wireless sensor networks with the help of the LEACH protocol. LEACH's stochastic cluster head selects an algorithm by a deterministic component to define the lifetime of a sensor network. It presents the three metrics - First Node Dies (FND), half of the Nodes Alive (HNA), and the Last Node Dies (LND) that determines the lifetime of a sensor network. However, it is assumed that all nodes in the network are homogenous and energy-constrained and are able to reach the base station, nodes have no location information, and cluster heads perform data compression.

Vasanth et al. [12] proposed an energy efficient routing for Virtual Back Bone Nodes (VBS) in which it maximizes the node life and turns off its radio when they are in sleep mode, in order to consume less energy. A concept of Restricted Back Bone Neighborhood Routing is proposed, which assures the efficient routing with minimum energy consumption of nodes and also implemented the critical transmission radius for Backbone nodes. An efficient method based on low energy consumption scheduling has to be implemented so as to schedule the node activity between sleep and active state. One solution isto create a backbone node and make their radios off when they are in sleep mode and the routing should be energy efficient by making the network life time prolong.

Arabi et al. [13] proposed HERF: A hybrid energy efficient routing using a fuzzy method in Wireless Sensor Networks. Authors work giving attention on Data broadcasting is a significant task performed by WSNs. The algorithms of this system depend on a number of factors such as application areas, practice condition, power, and aggregation factors. With respect to these parameters, various algorithms are recommended. An algorithm for hybrid energy efficient routing in wireless sensor networks, which used two algorithms, i.e. EF-Tree (Earliest-First Tree) and SID

(Source-Initiated Dissemination) to disseminate data, and employs a fuzzy method to choose group head, and to toggle between two methods, SID and EF-Tree.

Wei et al. [14] proposed an Energy-Efficient Wake-Up Scheduling for Data Collection and Aggregation. A sensor in wireless sensor networks periodically produces data as it monitors its area. The fundamental operation in such a network is the systematic gathering (with or without innetwork aggregation) and transmitting of sensed data to a base station for further processing. A key major challenge in WSNs is to schedule nodes' activities to reduce energy consumption.

Dhavachelvan et al.[15] worked for Energy-constrained WSN has attained considerable research concentration now days and requires robust and energy efficient routing protocols for communication in fading environments to minimize the energy consumption. To moderate the fading effects in the wireless channels, MIMO method is utilized for energy efficient communication system and to route the data in WSN. The cluster head nodes can cooperate the transmit data cooperatively before selecting the cooperative sending and receiving groups in each cluster. The theory has been used to elect healthier cluster heads having enough residual energy and high faith level. The theory has been used to select the cooperative nodes for MIMO communication. The outcome show that the CH-C-TEEM routing algorithm provides more than $50 \%$ increase in residual energy as compared to TEEM.

Jang et al. [16] worked on the development of the Wireless Sensor Network technology, ubiquitous technology comes to the fore as the core technology in the future. In the WSN, energy efficiency of the whole network is a key problem that has to be solved. Clustering is one of routing methods to improve energy efficiency. LEACH and LEACH-C are existing methodologies focused on optimizing energy efficiency of the network by applying clustering. Author suggest a new method, "Energy-Efficient Clustering scheme with Concentric Hierarchy (EECCH)," a centralized clustering scheme aimed at overcoming weaknesses of 
LEACH and LEACH-C both. By drawing circles with the base station as its center, the base point separates network nodes into some levels. The clusters have different numbers of its member nodes to eliminate inequality in energy dissipation through this process; it becomes possible to improve energy efficiency. By using MATLAB,

\section{ENERGY EFFICIENT ROUTING TECHNIQUE IN WSNS}

Minimization of energy consumption is serious issue in wireless sensor network. Various algorithm and techniques has been proposed and implemented for prolong routing. In this some of them is describing below:

\subsection{LEACH (Low Energy Adaptive Clustering Hierarchy)}

In the network, for distributing energy loads among the sensor nodes this adaptive clustering protocol is used. It uses single hop routing mechanism where information is transmitted directly to the cluster head or the sink [5]. It works in two phase

1) The setup phase: Organization of clusters, selection of cluster head is done and to determine whether a node can become a cluster head or not, an algorithm is used in each round.

2) The steady state phase: In order to minimize overhead the data is sent to the base station, the duration of the steady state phase is longer than the duration of the setup phase in order to minimize overhead. Cluster head creates a TDMA (Time Division Multiple Access) schedule based on the number of nodes in the group. CDMA (Code Division Multiple Access) code is used for random communication inside the cluster. LEACH is not suitable for large network areas.

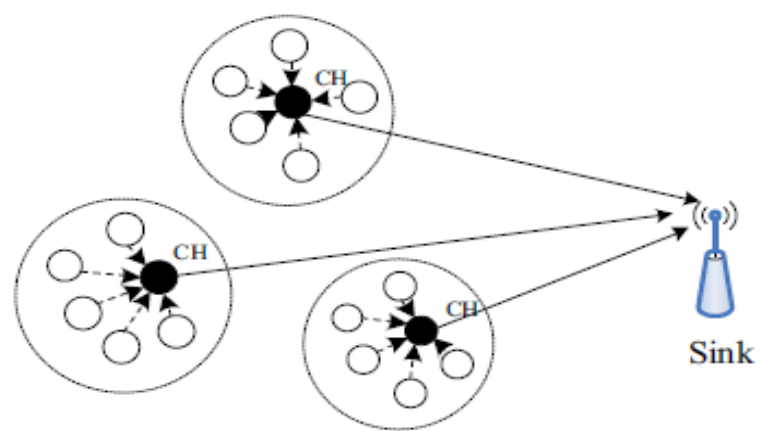

Fig.6: Leach cluster node

\section{Advantages}

1. It provides scalability in the network by means of limiting most of the communication inside the different clusters of the network.

2. It does not require the information of location of the sensor nodes in the network to create the clusters. Therefore it is very powerful routing protocol and it is very much simple also.

\section{Disadvantages}

1. It significantly relies on cluster heads rather than cluster members of the cluster for communicating to the sink. Due to this it incurs robustness issues like failure of the cluster heads.

2. In LEACH CHs are not uniformly distributed within the cluster that means $\mathrm{CHs}$ can be located at the edges of the cluster.

\subsection{Power-Efficient Gathering in Sensor Information Systems (PEGASIS):}

In [18], an enhancement over LEACH protocol was proposed. The protocol, called Power-Efficient Gathering in Sensor Information Systems (PEGASIS), is a near optimal chainbased protocol. The basic idea of the protocol is that in order to extend network lifetime, nodes need only communicate with their closest neighbors and they take turns in communicating with the base-station. When the round of all nodes communicating with the base-station ends, a new round will start and so on. This reduces the power required to transmit data per round as the power draining is spread uniformly over all nodes. Hence, PEGASIS has two main objectives. First, increase the lifetime of each node by using collaborative techniques and as a result the network lifetime will be increased. Second, allow only local coordination between nodes that are close together so that the bandwidth consumed in communication is reduced. Unlike LEACH, PEGASIS avoids cluster formation and uses only one node in a chain to transmit to the BS instead of using multiple nodes.

\section{Advantages}

1. Instead of forming multiple clusters, PEAGSIS construct a node chain when nodes are placed randomly in a play field then each node communicates only with a close neighbor and takes turns transmitting to the basestation, thus reducing the amount of energy spent per round.

2. It performs sound in the circumstances like unexpected variations in the identified characteristics like temperature.

\subsection{SPIN (Sensor Protocols for Information via Negotiation)}

In [19] the authors present a family of adaptive protocols, called SPIN (Sensor Protocols for Information via Negotiation), that efficiently disseminate information among sensors in an energy-constrained wireless sensor network. Nodes running a SPIN communication protocol name their data using high-level data descriptors, called meta-data. They use meta-data negotiations to eliminate the transmission of redundant data throughout the network. In addition, SPIN nodes can base their communication decisions both upon application-specific knowledge of the data and upon knowledge of the resources that are available to them. This allows the sensors to efficiently distribute data given a limited energy supply. Four specific SPIN protocols were simulated and analyzed: SPIN-PP and SPIN-EC, which are optimized for a point-to-point network, and SPIN-BC and SPIN-RL, which are optimized for a broadcast network.

\section{Advantages}

1. It Provide the resource awareness effectively

2. It enhances the availability of Metadata.

3. The duration of steady-phase is longer than the setup phase to minimize overhead

4. The nodes are only required to know its 1-hop neighborhood.

\section{Disadvantages}

1. It does not provide optimal routing.

2. It is not sure about the data will certainly reach the target or not and it is also not good for high-density distribution of nodes.

3. If the nodes that are interested in the data are far away from the source node and the nodes between 
source and destination are not interested in that data, such data will not be delivered to the destination at all.

4. It only compares flooding \& gossiping. It assumes that all nodes are interested in Data.

\subsection{TEEN (Threshold sensitive energy efficient sensor network protocol)}

For reactive network the first developed protocol was TEEN. The reduction of number of transmission is the purpose of hard threshold, which is done by allowing the nodes to transmit only when the sensed attribute is in the range of interest. The soft threshold further reduces the number of transmissions by eliminating all the transmissions which might have otherwise occurred when there is little or no change in the sensed attribute once the hard threshold. TEEN is well suited for time critical applications and is also quite efficient in terms of energy consumption and response time. It also allows the user to control the energy consumption and accuracy to suit the application. The main drawback of this scheme is if the thresholds are not achieved, the nodes will never communicate, the user will not get any data packet from the network and will not come to know about the nodes if they die. Thus, this scheme is not well suitable for applications where the user wants to get data regularly. Another problem is that a practical implementation would have to ensure that there collision-free cluster [20].

\section{Advantages}

1. This protocol is suitable for time critical applications and is also quite efficient in terms of energy consumption and response time. It also allows the user to control the energy consumption and accuracy to suit the application

2. It works well in conditions where sudden changes in the sensed attributes occur

\section{Disadvantages}

1. If the thresholds are not achieved, the nodes will never communicate; the user will not get any data packet from the network and will not come to know about the nodes if they die.

2. TEEN inclines to consume a lot of energy, because of long remoteness broadcasts.

3. When the number of covers rises, the broadcasts converts into shorter and overhead in the system stage as well as the process of the system exist

\subsection{SAR (Sequential Assignment Routing)}

SAR [21] is one of the first protocols for wireless sensor networks that provide the notion of QoS routing criteria. It is based on the association of a priority level to each packet. Additionally, the links and the routes are related to a metric that characterizes their potential provision of quality of service. This metric is based on the delay and the energy cost. Then, the algorithm creates trees rooted at the one-hop neighbors of the sink. To do so, several parameters such as the packet priority, the energy resources and the QoS metrics are taken into account. The protocol must periodically recalculate the routes to be prepared in case of failure of one of the active nodes.

\section{Advantages}

1. This protocol effective for fault tolerance

2. This protocol ensures for the data recovery more efficiently

\section{Disadvantages}

1. This protocol consumes more power

2. Also provides more overhead

3. It has Limited scalability properties

\subsection{GAF (Geographic Adaptive Fidelity)}

This protocol aims at optimizing the performance of wireless sensor networks by identifying equivalent nodes with respect to forwarding packets [22]. Two nodes are considered to be equivalent when they maintain the same set of neighbor nodes and so they can belong to the same communication routes. Source and destination in the application are excluded from this characterization. To identify equivalent nodes, their positions are necessary. Additionally, a virtual grid is constructed. This grid is formed by cells whose size allows to state that all the nodes in one cell can directly communicate with the nodes belonging to adjacent cells and vice versa. In this way, the nodes in a cell are equivalent. Nodes identify equivalent nodes by the periodic exchange of discovery messages with the nodes in their cells. With the information contained in these messages, the nodes negotiate which one is going to support the communications. The other nodes will stay powered off. With this procedure, the routing fidelity is kept, that is, there is uninterrupted connectivity between communicating nodes. However, the elected node periodically rotates for fair energy consumption. To do so, the nodes wake up periodically.

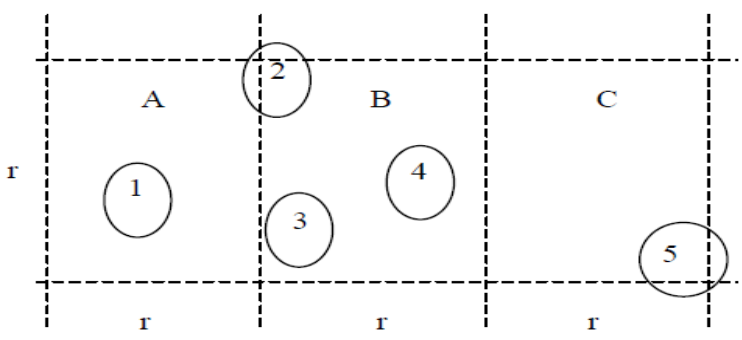

Fig. 7: An example of virtual grip in GAF

\section{Advantages}

1. It conserves energy by turning off unnecessary nodes in the network without affecting the level of routing fidelity

2. at least as well as a normal ad hoc routing protocol in terms of latency and packet loss and increases the lifetime of the network by saving energy

\section{Disadvantages}

1. This has less power awareness protocol

2. This does not provide data aggregation characteristic

\subsection{Directed Diffusion}

Directed Diffusion [23] is an important milestone in the datacentricrouting research of sensor networks. The idea aims at diffusing data through sensor nodes byusing a naming scheme for the data. The main reason behind using such a scheme is to get rid ofunnecessary operations of network layer routing in order to save energy. Direct Diffusionsuggests the use of attribute-value pairs for the data and queries the sensors in an on demandbasis by using those pairs. In order to create a query, an interest is defined using a list ofattribute-value pairs such as name of objects, interval, duration, geographical area, etc. The interest is broadcast by a sink through its neighbors. Each node receiving the interest can do caching for later use. The nodes also have the ability to do in-network data 
aggregation, which is modeled as a minimum Steiner tree problem. The interests in the caches are then used to compare the received data with the values in the interests. The interest entry also contains several gradient fields. A gradient is a reply link to a neighbor from which the interest was received. It is characterized by the data rate, duration and expiration time derived from the received interest's fields. Hence, by utilizing interest and gradients, paths are established between sink and sources. Several paths can be established so that one of them is selected by reinforcement. The sink resends the original interest message through the selected path with a smaller interval hence reinforces the source node on that path to send data more frequently. Fig.8, redrawn from, summarizes the Directed Diffusion protocol.

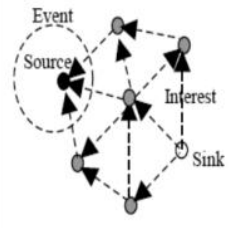

(a) Interest propagation

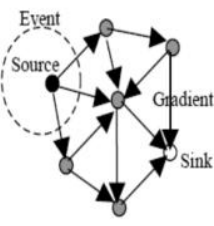

(b) Initial gradients setup

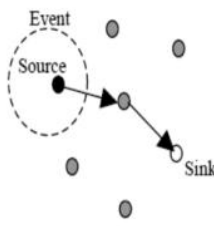

(c) Data delivery along reinforced
Fig.8: Directed diffusion protocol phases

\section{Advantages}

1. It is highly energy efficient since it is on demand and there is no need for maintaining global network topology.

2. It is designed to retrieve data aggregates from a single node.

3. Unlike other routing algorithms, in Directed Diffusion more than one sink can make queries and receive data at the same time; hence, simultaneous queries could be handled inside a single network.

\section{Disadvantages}

1. This protocol is application restricted and applicable for all applications

2. In addition, the naming schemes used in Directed Diffusion are application dependent and each time should be defined a priori. Moreover, the matching process for data and queries might require some extra overhead at the sensors.

3. An overhead problem occurs at the sensors during the matching process for data and queries

\section{CONCLUSION}

Energy conservation in wireless sensor network is serious issue because each sensor nodes of such network is battery oriented. During the transmission or reception of the packets the lifetime of the battery decreases and after certain time it will be dead. So to keep the battery active for long time the selection of the efficient routing is very essential due to which the energy and power of the sensor nodes can be improve. In this paper, we reviewed the earlier work done for the energy efficient routing by different authors and we also discuss some wireless sensor network routing protocols with their advantages and disadvantages. After review, it is found that some protocol is not much effective to conserve the energy and power of the sensor nodes so the design of efficient energy and power conserving protocol becomes so essential. In future work, need to design hybrid routing protocol which uses the best among the feature of the exiting routing protocol.

\section{REFERENCES}

[1] Maya M. Warrier, Ajay Kumar, "An energy efficient approach for routing in wireless sensor Networks", Global Colloquium in Recent Advancement and Effectual Researches in Engineering, Science and Technology (RAEREST 2016), Procedia Technology 25 ( 2016 ) 520 - 527, Elsevier.

[2] Yick, Jennifer, Biswanath Mukherjee, and Dipak Ghosal "Wireless sensor network survey" Computer NetworksElsevier52.12 (2008); 2292-2330.

[3] RafeAlasem, Ahmed Reda and Mahmud Mansour "Location based energy efficient reliable routing protocol for wireless sensor network "World Scientific and Engineering Academy ANDC Society(WSEAS) Stevens Point, Wisconsin, USA,2011.

[4] Mohammad S. Al_ Fares, Zhili Sun and Haitham Cruickshank, "A Hierarchical Routing Protocols for Survivability in Wireless Sensor Network" Proceedings of the International Multi Conference of Engineers and Computer Scientists 2009VolI, March 18-20, 2009, Hong Kong.

[5] Ivan Stojmenovic and Stephan Olariu. "Handbook of Sensor Networks: Algorithms and Architectures" in 2005.

[6] Luis Javier García Villalba, Ana Lucila Sandoval Orozco, Alicia Triviño Cabrera and Cláudia Jacy Barenco Abbas ,"Routing Protocols in Wireless Sensor Networks", Sensors 2009, 9, 8399-8421; doi:10.3390/s91108399.

[7] Hiba Sami Bazzi, Ahmad Bilal and Ali Masoud Haidar "Classification of routing protocols in wireless sensorNetwork", In proceeding of IEEE-2015.

[8] Sungoh Kwon and Ness B. Shroff "Energy-Efficient SINR-Based Routing for Multi-hop Wireless Networks," IEEE Transactions On Mobile Computing, VOL. 8, NO 5, MAY 2009.

[9] Chien-Erh Weng, Vishal Sharma, Hsing-Chung Chen and Chuan-Hsien Mao "PEER: Proximity-Based EnergyEfficient Routing Algorithm for Wireless Sensor Networks", Journal of Internet Services and Information Security (JISIS), volume: 6, number: 1 (February 2016), pp. $47-56$.

[10] Abdul Razaque, Musbah Abdulgader, Chaitrali Joshi, Fathi Amsaad, Mrunal Chauhan "P-LEACH: Energy Efficient Routing Protocol for Wireless Sensor Networks", In proceeding of IEEE xplore 2016.

[11] Razaque A, Elleithy K. and Handy, "Modular energyefficient and robust paradigms for a disaster-recovery process over wireless sensor networks", Sensors. 2015 Jul 6; 15(7):16162-95.

[12] Umesh B.N, Dr G Vasanth and Dr Siddaraju "Energy Efficient Routing of Wireless Sensor Networks Using Virtual Backbone and life time Maximization of Nodes", International Journal of Wireless \& Mobile Networks (IJWMN) Vol. 5, No. 1, February 2013.

[13] Yanwei Wu, Xiang-yang Li, Mo Li, Wei Lou, EnergyEfficient Wake- Up Scheduling for Data Collection and Aggregation, Parallel and Distributed Systems, IEEE Transactions on , vol.21, no.2, pp.275,287, Feb. 2010 
[14] Arabi, Z., HERF: A hybrid energy efficient routing using a fuzzy method in Wireless Sensor Networks, Intelligent and Advanced Systems (ICIAS), 2010 International Conference on , vol., no., pp.1,6, 15-17 June 2010.

[15] Sathian, D. Baskaran, R.; Dhavachelvan, P., "Lifetime enhancement by Cluster Head Cooperative Trustworthy Energy Efficient MIMO routing algorithm based on game theory for WSN," Computing Communication \& Networking Technologies (ICCCNT), 2012 Third International Conference on , vol., no., pp.1,5, 26-28 July 2012.

[16] Seongsoo Jang; Ho-Yeon Kim; Nam-Uk Kim; TaiMyoung Chung, "Energy-Efficient Clustering scheme with Concentric Hierarchy," RF and Microwave Conference (RFM), 2011 IEEE International , vol., no., pp.79,82, 12-14 Dec. 2011.

[17] Jia Xu, Ning Jin, Xizhong Lou, Ting Peng, Qian Zhou, Yanmin Chen "Improvement of LEACH protocol for WSN", 2012, IEEE.

[18] S. Lindsey, C.Raghavendra, "PEGASIS: Power-Efficient Gathering in Sensor Information Systems", IEEE
Aerospace Conference Proceedings, 2002, Vol. 3, 9-16 pp. 1125-1130.

[19] Kulik, J.; Heinzelman, W.; Balakrishnan, H. Negotiationbased Protocols for Disseminating Information in Wireless Sensor Networks. Wirel. Netw.2002, 8, 169185 .

[20] Pallavi S. Katkar et al, / (IJCSIT) International Journal of Computer Science and Information Technologies, Vol. 6 , 2015.

[21] Al-Karakin, J.N.; Kamal, A.E. Routing Techniques in Wireless Sensor Networks: A Survey. IEEE Wirel. Commun. 2004, 11, 6-28.

[22] Xu, Y.; Heidemann, J.; Estrin, D. Geography-informed Energy Conservation for $\mathrm{Ad} H \mathrm{Hoc}$ Routing. In Proceedings of the Seventh Annual International Conference on Mobile Computing and Networking (MOBICOM), Rome, Italy, July, 2001; pp. 70-84.

[23] Kemal Akkaya and Mohamed Younis "A Survey on Routing Protocols for Wireless Sensor Networks", Elsevier 2005. 